\title{
Parameterised models for the lensing cluster Abell 1689
}

\author{
L. J. King ${ }^{1,2}$, D. I. Clowe ${ }^{2}$, and P. Schneider ${ }^{2,1}$ \\ 1 Max-Planck-Institut für Astrophysik, Karl-Schwarzschild Str 1, Garching bei München, Germany \\ 2 Institut für Astrophysik und Extraterrestrische Forschung der Universität Bonn, Auf dem Hügel 71, \\ 53121 Bonn, Germany
}

Received 20 July 2001 / Accepted 3 December 2001

\begin{abstract}
Here we apply a recently developed maximum likelihood method for determining best-fit parameterised lens models to observations of the rich lensing cluster Abell 1689. The observations that we use were taken with the ESO/MPG Wide Field Imager. The wide field-of-view enables us to use the weakly lensed images of faint background objects on an unsurpassed range of scales, $0.12 h^{-1} \mathrm{Mpc}<R<1.8 h^{-1} \mathrm{Mpc}$ from the cluster centre, to determine best-fit models for the 1-parameter singular isothermal sphere (SIS), 2-parameter general power-law and NFW models, and 3-parameter singular isothermal ellipsoid (SIE). The best-fit SIS has an Einstein radius $\theta_{\mathrm{E}}=0^{\prime} .37\left(0.043 h^{-1} \mathrm{Mpc}\right)$ i.e. a velocity dispersion $\sigma_{1 \mathrm{D}}=1028_{-42}^{+35} \mathrm{~km} \mathrm{~s}^{-1}$ in an $\Omega=1.0, \Lambda=0.0$ cosmology. For the best-fit NFW profile, the virial radius $r_{200}=1.14 h^{-1} \mathrm{Mpc}$ and the concentration parameter $c=4.7$, giving a virial mass $M_{200}=5.7 \times 10^{14} h^{-1} M_{\odot}$. At $q=0.88$, the slope of the best-fit power-law model is slightly flatter than isothermal $(q=1.0)$, indicating that the galaxies most important in the fitting procedure lie inside the scale radius $r_{\mathrm{s}}$. By fitting an SIE, the deviation of the projected mass distribution from circular symmetry is evident, with a best-fit axial ratio $f=0.74$.
\end{abstract}

Key words. dark matter - gravitational lensing - large-scale structure of Universe - galaxies: clusters: general methods: statistical

\section{Introduction}

The gravitational field of a cluster acting as a weak lens distorts the shapes of background galaxies (shear effect), and changes their observed number density (magnification effect); see Mellier (1999) and Bartelmann \& Schneider (2001) for recent reviews. These imprints on the background galaxy population can be used to constrain the projected mass distribution of the cluster. Parameterised cluster models are essential when statistical comparisons of clusters are to be made, and their error properties are well understood in contrast to those of non-parametric mass maps.

In Schneider et al. (2000; hereafter SKE) we developed likelihood techniques to obtain parameterised models from weak lensing data, and quantified the accuracy with which parameters can be recovered using the magnification and/or shear information. This work concentrated on power-law models, and was extended in King \& Schneider (2001; hereafter KS) to encompass NFW (Navarro et al. 1996, 1997) and ellipsoidal models (Kormann et al. 1994),

Send offprint requests to: L. J. King,

e-mail: lindsay@astro.uni-bonn.de also investigating the effect of uncertainty in the redshifts of potential source galaxies. In King et al. (2001; hereafter KSS) we considered the influence of substructure, concluding that the method is robust against the presence of substructure at a level consistent with $N$-body simulations. Gray et al. (2000) applied the magnification maximum likelihood method to near-infrared CIRSI observations of the cluster Abell 2219, and extended it to include the effect of incompleteness.

In this paper we obtain parameterised models of a lensing cluster by applying the shear maximum likelihood method. We use WFI observations of the rich $z_{\mathrm{d}}=0.18$ lensing cluster Abell 1689, the observational details of which are presented in Clowe \& Schneider (2001).

The families of lens models considered are the singular isothermal sphere (SIS), the singular isothermal ellipsoid (SIE), a general power-law model and the NFW profile. The SIS model is frequently used as the basis model for lensing studies, and the introduction of an ellipticity, yielding the SIE model, allows us to investigate nonspherically symmetric dark matter distributions. The motivation for considering the NFW profile is that it is a good description of the radial density profiles of virialised 
dark matter halos formed in cosmological simulations of hierarchical clustering. We also investigate a general single slope power-law model, since in KSS we found that lensing simulations through an $N$-body cluster were well fit by this model.

The structure of this work is as follows: in Sect. 2 we summarise how the shear information is incorporated into a likelihood analysis, and outline the relevant observational parameters. Our results are presented in Sect. 3, and we finish with a discussion and conclusions in Sect. 4 .

\section{An outline of the shear method and observational details}

The notation and basic relationships used in this paper are as in KS, and the reader is referred to SKE for details of the maximum likelihood treatment. Throughout, standard lensing notation is used (e.g. Schneider et al. 1992; Bartelmann \& Schneider 2001).

\subsection{The shear method}

The basis of using the distorted images of background galaxies to constrain the cluster model is that the expectation value for the lensed ellipticity $\langle\epsilon\rangle=g$, the reduced shear, in the non-critical regime (e.g. Schramm \& Kayser 1995), and that $\langle\epsilon\rangle=1 / g^{*}$ in the critical regime (Seitz \& Schneider 1997).

A parameterised model specifies the dependence of $g(\boldsymbol{\vartheta})$ on position $\boldsymbol{\vartheta}$ for a profile family; these are given in Sect. 2.3 below, for the families considered. Minimising the shear log-likelihood function (see SKE for details)

$\ell_{\gamma}=-\sum_{i=1}^{N_{\gamma}} \ln p_{\epsilon}\left(\epsilon_{i} \mid g\left(\boldsymbol{\vartheta}_{i}\right)\right)$

over the $N_{\gamma}$ galaxy images gives $\pi_{\max }$, the parameters most consistent with the probability distribution of lensed ellipticities $p_{\epsilon}(\epsilon \mid g(\boldsymbol{\vartheta}))$. The trial parameters en route to the best-fit will be referred to by $\pi$. Noise in this method arises from the intrinsic dispersion in the galaxy ellipticity distribution, $\sigma_{\epsilon^{\mathrm{s}}}$, and observational effects.

\subsection{Observational parameters}

The catalogue of lensed galaxies in the cluster A1689 containing positions $\boldsymbol{\vartheta}_{i}$ and lensed ellipticities $\epsilon_{i}$ is described in Clowe \& Schneider (2001). Objects therein were selected to have $23<m_{R}<25.5$, and a signal-to-noise greater than 9 . The data field used in the method has inner and outer radii of $\theta_{\text {in }}=1^{\prime} .0\left(0.12 h^{-1} \mathrm{Mpc}\right)$ and $\theta_{\text {out }}=15^{\prime} .4$ $\left(1.8 h^{-1} \mathrm{Mpc}\right)$ respectively. Within this aperture, there are $N_{\gamma}=19400$ images for which an ellipticity $\epsilon_{i}$ can be measured, and can therefore be used for the shear method. These galaxies have a Gaussian probability distribution with 2-D dispersion $\sigma_{\epsilon}=0.424$; since $|g| \lesssim 0.2$, correction to the unlensed dispersion is not necessary.
The redshift distribution of the source galaxy population can safely be neglected, since the lens is at a fairly low redshift $\left(z_{\mathrm{d}}=0.18\right)$ (see for example Bartelmann \& Schneider 2001). This amounts to approximating the background galaxies to be located at a redshift corresponding to the mean value of their lensing effectiveness parameter $w(z)$. If the cluster was at a higher redshift, $\gtrsim 0.25$ say, then the redshift distribution of the galaxies would become important and this sheet approximation is no longer robust. We take the background sources to be at $z=1.0$ unless otherwise stated. This is motivated by Clowe \& Schneider (2001), who used the HDF-South photometric redshift catalogue from Fontana et al. (1999) and found a mean galaxy redshift of 1.15 after applying the same magnitude cuts as above. Since this value is affected by Poisson noise and cosmic variance, being measured from only 48 galaxies, we consider the influence of the assumed source redshift in Sect. 3.4. Unless otherwise stated, we give the results for an $\Omega=1.0, \Lambda=0.0$ cosmology, for a Hubble constant $H_{0}=100 h \mathrm{~km} \mathrm{~s}^{-1} \mathrm{Mpc}^{-1}$. At $z_{\mathrm{d}}=0.18$, an angular scale of $1^{\prime} .0$ corresponds to a linear scale of $0.12 h^{-1} \mathrm{Mpc}$.

\subsection{Profiles}

\subsubsection{The isothermal profile}

The singular isothermal profile, or SIS, is characterised by a velocity dispersion $\sigma_{1 \mathrm{D}}$ that is constant with radius. It is parameterised by an Einstein radius

$\theta_{\mathrm{E}}=\frac{4 \pi \sigma_{1 \mathrm{D}}^{2}}{c^{2}} \frac{D_{\mathrm{ds}}}{D_{\mathrm{s}}}$

which marks the boundary between the strong and weak lensing regimes. Note that for a given $\sigma_{1 \mathrm{D}}$, the Einstein radius depends on $\Omega$ and $\Lambda$ through the angular diameter distances, but that it is independent of $H_{0}$. The surface mass density is given by

$\kappa(\theta)=0.5\left(\frac{\theta}{\theta_{\mathrm{E}}}\right)^{-1}$.

The mean surface mass density inside $\theta$ is $\bar{\kappa}(\theta)=2 \kappa(\theta)$, and further $|\gamma|=\kappa$.

\subsubsection{The singular isothermal ellipsoidal profile}

The SIE profile is characterised by a velocity dispersion that is constant with radius; it is parameterised by an equivalent angular Einstein radius $\theta_{\mathrm{E}}$, an axial ratio $0<f \leq 1$ and an orientation $\alpha$. Let the polar coordinates in the lens plane be $\boldsymbol{\vartheta}=(\theta \cos \phi, \theta \sin \phi)$. The equivalent angular Einstein radius for a lens with velocity dispersion $\sigma_{1 \mathrm{D}}$ is given by Eq. (2). The dimensionless surface mass density is given by

$$
\begin{aligned}
& \kappa(\theta, \phi)=\frac{\sqrt{f}}{2 b} \\
& b=\frac{\theta}{\sqrt{2} \theta_{\mathrm{E}}}\left(\left(1+f^{2}\right)+\left(1-f^{2}\right) \cos [2(\phi-\alpha)]\right)^{\frac{1}{2}}
\end{aligned}
$$


and the magnification is

$\mu(\theta, \phi)=\frac{1}{1-2 \kappa(\theta, \phi)}$.

The components of the shear are

$\gamma_{1}=-\kappa \cos (2 \phi) ; \quad \gamma_{2}=-\kappa \sin (2 \phi)$.

\subsubsection{The power-law profile}

The power-law profile can be characterised by an Einstein radius $\theta_{\mathrm{E}}$ and a slope $q$, such that

$\kappa(\theta)=\frac{2-q}{2}\left(\frac{\theta}{\theta_{\mathrm{E}}}\right)^{-q}$,

and

$\bar{\kappa}(\theta)=\left(\frac{\theta}{\theta_{\mathrm{E}}}\right)^{-q}$.

The shear is

$\gamma(\theta)=\frac{q}{2}\left(\frac{\theta}{\theta_{\mathrm{E}}}\right)^{-q}$

In KSS we found that the power-law profile can provide almost as good a fit as the NFW profile, depending on the size of the cluster's scale radius $r_{\mathrm{s}}$ relative to the available data field.

\subsubsection{The NFW profile}

The properties of the NFW profile in the context of gravitational lensing have been discussed by several authors including Bartelmann (1996) and Wright \& Brainerd (2000). We can parameterise this profile with a virial radius $r_{200}$, and a dimensionless concentration parameter $c$, which are related through a scale radius $r_{\mathrm{s}}=r_{200} / c$. Inside $r_{200}$, the mass density of the halo equals $200 \rho_{\mathrm{c}}$, where $\rho_{\mathrm{c}}=\frac{3 H^{2}(z)}{8 \pi G}$ is the critical density of the Universe at the redshift of the halo. The characteristic overdensity of the halo, $\delta_{c}$, is related to $c$ through

$$
\delta_{\mathrm{c}}=\frac{200}{3} \frac{c^{3}}{\ln (1+c)+c /(1+c)} .
$$

Then the density profile is

$\rho(r)=\frac{\delta_{\mathrm{c}} \rho_{\mathrm{c}}}{\left(r / r_{\mathrm{s}}\right)\left(1+r / r_{\mathrm{s}}\right)^{2}}$,

which is shallower than isothermal $\left(r^{-2}\right.$ in 3 -D) near the halo centre and steeper than isothermal for $r \gtrsim r_{\mathrm{s}}$. For details of quantities required in the lensing analysis, see KS.

\section{Results}

\subsection{The best-fit parameterised models}

The shear method was applied to the catalogue of background objects, finding the best-fit model parameters, for each of the model families described above.
Table 1. The best-fit parameters $\pi_{\max }$ for the SIE, power-law (POW) and NFW profiles are presented in the middle column. The final column shows $2 \Delta \ell \equiv$ $2\left[\ell\left(\pi_{\max }(\right.\right.$ Model $\left.\left.)\right)-\ell\left(\pi_{\max }\left(\operatorname{Model}_{\mathrm{NFW}}\right)\right)\right]$.

\begin{tabular}{|l|l|l|}
\hline Model & Best-fit parameters $\pi_{\max }$ & $2 \Delta \ell$ \\
\hline SIE & $\theta_{\mathrm{E}}=0^{\prime} .37 ; \quad f=0.74 ; \alpha=-27^{\circ}$ & 2.5 \\
POW & $\theta_{\mathrm{E}}=0^{\prime} .3 ; \quad q=0.88 \quad$ & 3.2 \\
NFW & $r_{200}=1.14 h^{-1} \mathrm{Mpc} ; \quad c=4.7$ & - \\
\hline
\end{tabular}

For the 1-parameter SIS, the best-fit $\theta_{\mathrm{E}}=0^{\prime} .37$; this corresponds to a linear size of $0.043 h^{-1} \mathrm{Mpc}$ at the lens redshift. The values of $2 \Delta \ell \equiv 2\left[\ell(\pi)-\ell\left(\pi_{\max }\right)\right]$ were calculated from the data in order to obtain confidence levels for the fit. The upper (lower) 1- $\sigma$ limits are 0'395 (0'34) and the upper (lower) $3-\sigma$ limits are $0^{\prime} .44$ (0'.29). This translates into $\sigma_{1 \mathrm{D}}=1028_{-42}^{+35} \mathrm{~km} \mathrm{~s}^{-1}$ (1- $\sigma$ errors), for $\Omega=1.0$ and $\Lambda=0.0$. In an $\Omega=0.3, \Lambda=0.7$ Universe we obtain $\sigma_{1 \mathrm{D}}=998_{-42}^{+33} \mathrm{~km} \mathrm{~s}^{-1}(1-\sigma$ errors $)$; this is within the $1-\sigma$ error of the result given in Clowe \& Schneider (2001) for the same choice of cosmology and background source redshift, calculated with a direct $\chi^{2}$ fit to the shear profile.

In Table 1 , we present the values of $\pi_{\max }$ most consistent with the data for the 2-parameter power-law and NFW profiles, and for the 3-parameter SIE profile. Comparing the 2-parameter families, the overall best-fit model comes from the NFW family but it is only marginally preferred over the power-law model. For 2 degrees of freedom, the values of $2 \Delta \ell \equiv$ $2\left[\ell\left(\pi_{\max }(\right.\right.$ power - law $\left.\left.)\right)-\ell\left(\pi_{\max }(\mathrm{NFW})\right)\right]$ show that the best-fit models are only distinguished at about $80 \%$ confidence.

In order to fit the SIE profile, we adopted a complex representation for the ellipticity of the lensing cluster:

$\epsilon_{\mathrm{A} 1689}=\frac{1-f}{1+f} \mathrm{e}^{2 \mathrm{i} \alpha}$

and minimised $\ell$ in $\epsilon_{1}-\epsilon_{2}-\theta_{\mathrm{E}}$ parameter space. Fitting this profile shows that the data require an asphericity in the lensing mass distribution, which is also suggested both by the luminous mass distribution and by non-parametric mass reconstruction.

For the NFW model in an $\Omega=1.0, \Lambda=0.0$ Universe, confidence contours $\left(2 \Delta \ell=2\left[\ell(\pi)-\ell\left(\pi_{\max }\right)\right]\right)$ are shown in Fig. 1. Confidence contours for the power-law model are shown in Fig. 2.

Figure 3 shows the enclosed projected surface mass $M_{\Sigma}(<R)$ (in units of $M_{\odot}$ ) for each of the SIS, POW and NFW models, as a function of projected distance from the profile centre $R$ (in kpc) plotted out to $R=r_{200}=1.14 h^{-1} \mathrm{Mpc}$. Note the good agreement between the total masses estimated by fitting each of the models: the maximum difference beween the models is $\sim 20 \%$ at the virial radius and smaller for smaller radii. 


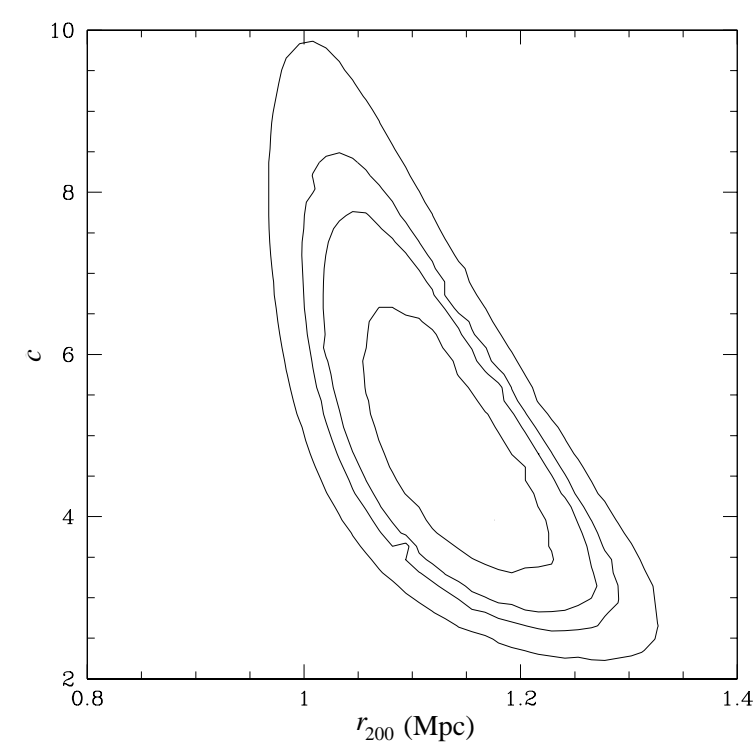

Fig. 1. This figure shows the confidence contours for the NFW profile family. We show contours of constant $2 \Delta \ell=$ $2\left[\ell(\pi)-\ell\left(\pi_{\max }\right)\right]$. Starting with the innermost contour, the levels are plotted at $68.3,90,95.4$ and $99 \%$ confidence.

\subsection{Assessing the goodness of fit}

Just how good a fit is the best-fit NFW model, $\pi_{\max }$ ? Specifying $g(\boldsymbol{\vartheta})$ for $\pi_{\max }$, the catalogue was "unlensed" by transforming the observed $\epsilon_{i}$ to intrinsic $\epsilon_{i}^{\mathrm{s}}$ under this lens model. Taking this reference catalogue, the phases of $\epsilon_{i}^{\mathrm{S}}$ were randomised, while preserving $\boldsymbol{\vartheta}_{i}$, and $\left|\epsilon_{i}^{\mathrm{s}}\right|$, to obtain a new catalogue. This catalogue was lensed and the value of $\ell_{R}\left(\pi_{\max }\right)$, where the subscript $R$ denotes randomisation, was determined for this realisation. This randomisation of the reference catalogue, lensing and determination of $\ell_{R}\left(\pi_{\max }\right)$ was repeated 10000 times, to build up statistics of $\Phi:=2\left[\ell_{R}\left(\pi_{\max }\right)-\ell\left(\pi_{\max }\right)\right]$; the cumulative probability distribution $P(<\Phi)$ is shown in Fig. 4 . The value $\ell\left(\pi_{\max }\right)$ (fit to the real data set) lies well within the distribution of $\ell_{R}\left(\pi_{\max }\right)$ for the random phase data sets, with 43.9 (56.1)\% of realisations being more (less) consistent with the model.

We repeated the process for the power-law model, and in this case, 44.3 (55.7)\% of randomised data sets are more (less) consistent with the model.

\subsection{The influence of the aperture size}

In order to test the influence of the size of the data field on the recovered NFW parameters, the value of $\theta_{\text {out }}$ was decreased from $15^{\prime} .4$ and $\pi_{\max }$ obtained for these catalogues. The largest deviation of $\pi_{\max }$ for the smaller apertures compared with the full aperture is at most $10 \%$ until $\theta_{\text {out }} \sim 8^{\prime} .0$. For instance, when $\theta_{\text {out }}=10^{\prime} .0$ the deviation of both $r_{200}$ and $c$ from their values when $\theta_{\text {out }}=15^{\prime} .4$ is well under $1 \%$. Below about $8^{\prime} .0$, the shear signal becomes noisy, and changing the aperture slightly can have

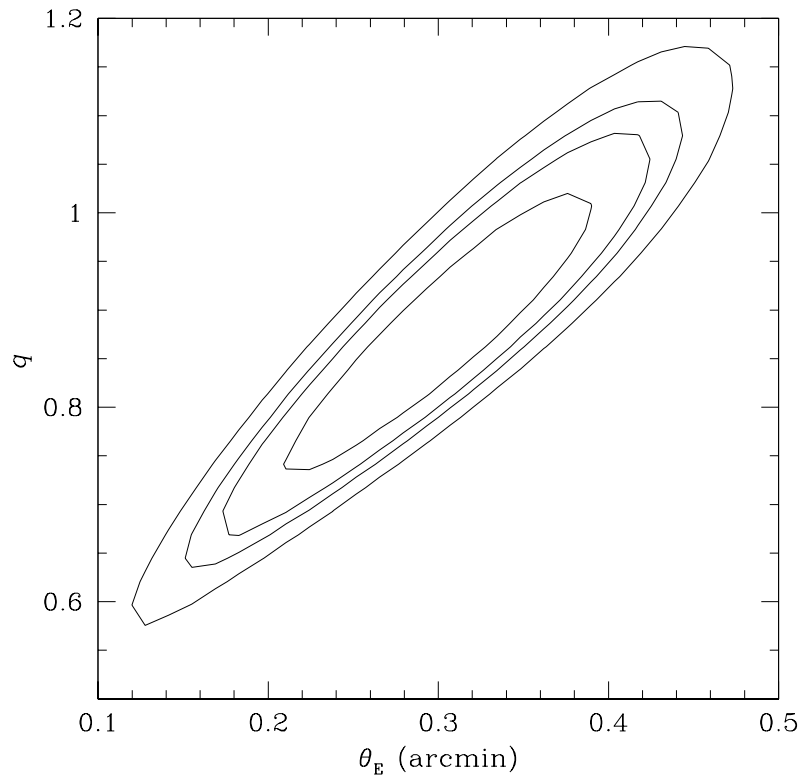

Fig. 2. Same as Fig. 1 for the power-law family.

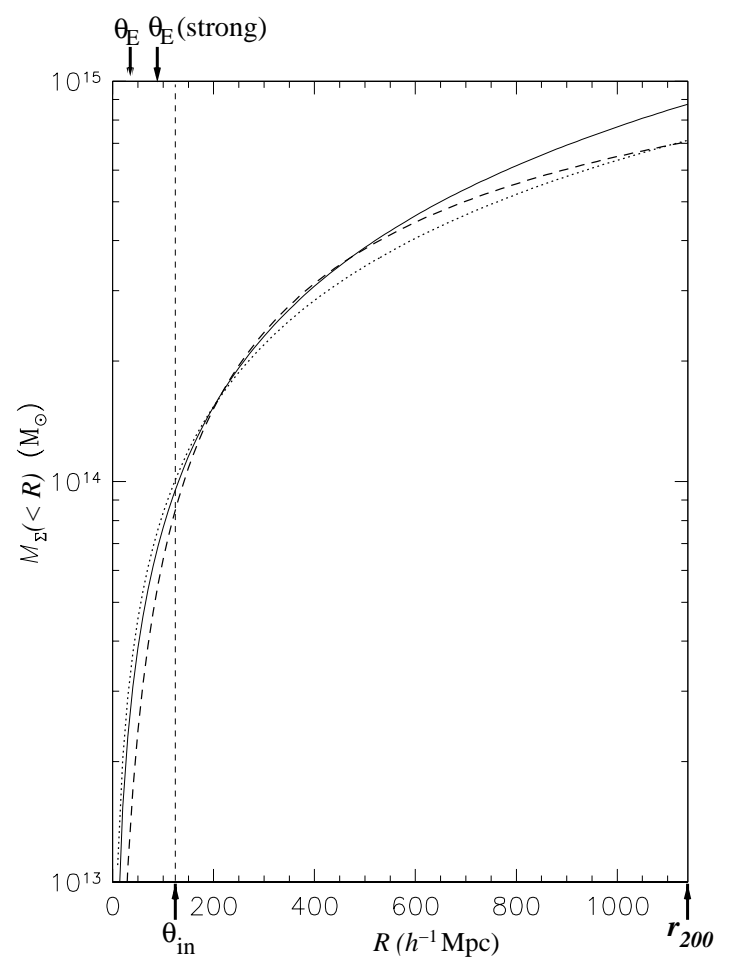

Fig. 3. The behaviour of the enclosed surface mass $M_{\Sigma}(<R)$ as a function of projected distance $R$ from the cluster centre, plotted out to $r_{200}=1.14 h^{-1} \mathrm{Mpc}$ for the best-fit SIS (solid line), POW (dotted line) and NFW (dashed line) models. The inner fitting radius, $\theta_{\text {in }}$ is indicated by a vertical dashed line (the outer fitting radius, $\theta_{\text {out }}$, is larger than $r_{200}$ ). On the upper horizontal axis, the best-fit SIS Einstein radius from weak lensing is denoted by $\theta_{\mathrm{E}}$, and the Einstein radius implied by the strong lensing arcs by $\theta_{\mathrm{E}}$ (strong).

a significant effect on parameter recovery. Another effect of decreasing aperture size is to expand the confidence 


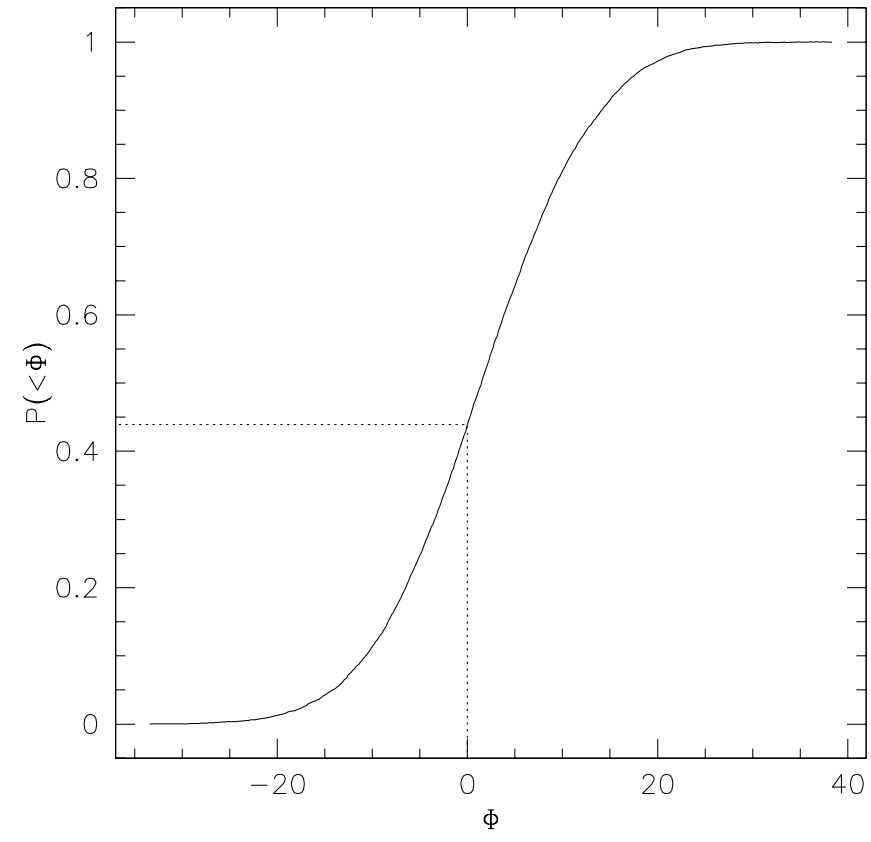

Fig. 4. Goodness of fit results for the NFW model: the distribution of $P(<\Phi)$ for $\Phi:=2\left[\ell_{R}\left(\pi_{\max }\right)-\ell\left(\pi_{\max }\right)\right]$ determined from 10000 randomisations of the background galaxy position angles.

contours of the best-fit model; this has been discussed in detail in SKE.

When $\theta_{\text {out }}=15^{\prime} .4$ and $\theta_{\text {in }}$ is increased from $1^{\prime} .0$ to $2^{\prime} .0$, the best-fit NFW model still lies within the original $68.3 \%$ confidence contour.

\subsection{The impact of background source redshift and cosmology}

Concentrating on the NFW profile to examine the impact of our choice of background source redshift and cosmology, Table 2 shows the values of $\pi_{\max }$ obtained with various assumptions. To facilitate the comparison between the different scenarios, we also give values for $M_{200}$, the mass contained within a sphere of radius $r_{200}$ and having mean density $200 \rho_{\mathrm{c}}$. Results are shown for an $\Omega=1.0, \Lambda=0.0$ cosmology, and for an $\Omega=0.3, \Lambda=0.7$ cosmology with $z=1$.

If the background sources are at $z=1.25$ rather than at $z=1.0, M_{200}$ would be about $5 \%$ lower, and if the sources were at $z=0.75$ the mass estimate would be about $10 \%$ higher. Working in an $\Omega=0.3, \Lambda=0.7$ cosmology, and keeping $z=1.0$, gives an increase of about $5 \%$ in the best-fit value of $M_{200}$. Again, we can compare this result with the value obtained by Clowe \& Schneider assuming the same cosmology and $z=1.0$ : the values of $r_{200}$ and $c$ in that work correspond to an $M_{200}$ value that is $2.5 \%$ higher than that obtained here.
Table 2. This table shows $\pi_{\max }$ and the corresponding value of $M_{200}$ for the NFW profile, assuming different redshifts for the background sources, $z$. An $\Omega=1.0, \Lambda=0.0$ Universe is denoted by EdS, and an $\Omega=0.3, \Lambda=0.7$ Universe by $\Lambda$-flat.

\begin{tabular}{|l|l|l|l|l|}
\hline Cosmology & $z$ & $\begin{array}{l}r_{200} \\
\left(h^{-1} \mathrm{Mpc}\right)\end{array}$ & $c$ & $\begin{array}{l}M_{200} \\
\left(10^{14} h^{-1} M_{\odot}\right)\end{array}$ \\
\hline EdS & 0.5 & 1.27 & 5.2 & 7.8 \\
EdS & 0.75 & 1.18 & 4.8 & 6.3 \\
EdS & 1.0 & 1.14 & 4.7 & 5.7 \\
EdS & 1.25 & 1.12 & 4.6 & 5.4 \\
\hline$\Lambda$-flat & 1.0 & 1.29 & 4.8 & 5.95 \\
\hline
\end{tabular}

\section{Discussion and conclusions}

The mass profile of a cluster can be constrained by using the weakly lensed images of background galaxies; since lensing does not depend on the nature of the matter, it is an invaluable means to study the luminous and dark matter. One technique that harnasses the infomation contained in the distorted ellipticities of the background population is the shear likelihood method developed in SKE and in KS. In this paper we have applied the method to WFI observations of the $z_{\mathrm{d}}=0.18$ lensing cluster Abell 1689, to obtain best-fit SIS, SIE, NFW and power-law models. Up until now, we had rigorously tested our method on synthetic data sets, generated by lensing through analytic profiles and numerically simulated clusters.

The maximum likelihood method developed in SKE and extended in KS differs from standard $\chi^{2}$ fitting (used recently by Clowe \& Schneider (2001) for example) in a few respects:

- Each galaxy is treated individually rather than being binned.

- The most likely model is found by finding the parameters that maximise the probabibility of the lensed ellipticity distribution. In $\chi^{2}$ fitting, the difference between the model and observed reduced shear is minimised, taking into account the observational errors.

- The unlensed ellipticity distribution is assumed to be a gaussian in the maximum likelihood method, whereas the lensed ellipticity distribution is assumed to be a gaussian in $\chi^{2}$ fitting.

Since a subset of the models considered here, namely the NFW and SIS models, were considered by Clowe \& Schneider (2001), we can directly compare the results.

The distorted images of background sources, at projected distances between $1^{\prime} .0$ and $15^{\prime} .4\left(0.12 h^{-1} \mathrm{Mpc}<\right.$ $R<1.8 h^{-1} \mathrm{Mpc}$ ) from the centre of the cluster have been used to obtain the best-fit models. In an $\Omega=1.0, \Lambda=0.0$ cosmology, the best-fit 1-parameter SIS model has $\sigma_{1 \mathrm{D}}=$ $1028_{-42}^{+35} \mathrm{~km} \mathrm{~s}^{-1}$. This velocity dispersion is consistent with that of the X-ray study of Allen (1998), who investigated 
a cluster sample and obtained temperature profiles by applying a deprojection technique to X-ray surface brightness profiles. Dynamical analyses of the cluster indicate that it is not a simple system: Teague et al. (1990) found $\sigma_{1 \mathrm{D}}=2355 \mathrm{~km} \mathrm{~s}^{-1}$, and evidence for three other groups of galaxies in its foreground. A more recent analysis by Girardi et al. (1997) finds that A1689 is composed of three structures for which the resultant $\sigma_{1 \mathrm{D}} \sim 560 \mathrm{~km} \mathrm{~s}^{-1}$ is much lower. Girardi et al. (1997) point out that their result, along with the foreground groups suggested by Teague et al. (1990), may indicate the presence of a filamentary structure along the line of sight. The strong lensing giant arcs imply an Einstein radius $\theta_{\mathrm{E}} \approx 0.75$ (e.g. Miralda-Escudé \& Babul 1995). Assuming the arcs are at $z=3$, the corresponding SIS has $\sigma_{1 \mathrm{D}}=1360 \mathrm{~km} \mathrm{~s}^{-1}$. In fact, $\sigma_{1 \mathrm{D}}$ is rather insensitive to $z$, decreasing by less than $2 \%$ even when $z=6$.

As discussed in Clowe \& Schneider (2001), this data set gives lower limits for the mass of the lensing cluster, due to contamination of the galaxy catalogue with stars and foreground and cluster dwarf galaxies. Even with any realistic correction considered, they find that the weak lensing value can not be reconciled with that interpreted from strong lensing data. This points to the need for more spectroscopic and photometric redshift information in both regimes, and a thorough combined analysis of the weak and strong lensing data.

Formally, the best-fit 2-parameter model belongs to the NFW family $\left(r_{200}=1.14 h^{-1} \mathrm{Mpc}, c=4.7\right)$, but it cannot be distinguished from the other best-fit models at the 3- $\sigma$ level. In order to distinguish profiles at a satisfactory significance, so testing the CDM paradigm (that predicts NFW and similar cuspy profiles) stacking the signals from a number of clusters is required.

The best-fit general power-law has a slope $q=0.88$, and $\theta_{\mathrm{E}}=0.3\left(0.035 h^{-1} \mathrm{Mpc}\right)$. One might have expected a steeper slope, since most of the background galaxies sample the profile at radii outside $r_{\mathrm{s}}$, where the slope is expected to be steeper than isothermal. Our result indicates that galaxies close to $\theta_{\text {in }}$ are important in the determination of the best-fit model.

The confidence contours for the NFW result show that $r_{200}$ is better constrained than $c$, consistent with our study of NFW profiles in KS. Thus $M_{200}$, which depends on $r_{200}$, is also well constrained. When the power-law is fit, the confidence contours show a smaller fractional uncertainty in $q$ than in $\theta_{\mathrm{E}}$, which is in agreement with our finding in SKE.

We also considered a 3-parameter SIE model; this model reveals that the projected (dark and luminous) surface mass density is not circularly symmetric. The appearance of the cluster on optical images, and a direct non-parametric mass reconstruction also suggest deviation from circular symmetery. It is interesting that although the SIE is a 3-parameter model, its likelihood is in fact lower than that of the 2-parameter NFW model. This suggests that in order to fit the weak lensing data, any deviation from circular symmetry is not as important a driver as the deviation from a purely isothermal profile slope. Another factor coming into play may also be the "twisting" of the isodensity contours as a function of distance from the centre; this is evident when the fitting aperture is changed, and even directly on the non-parametric reconstruction.

By randomising the phases of the background sources, we tested that the NFW model and power-law models are indeed good fits to the real data. One point to note is that the galaxy catalogue must be unlensed before the phases are randomised and the catalogue is again lensed. If the catalogue is not unlensed, this results in a significant offset between the likelihood values from these randomisations compared with the maximum likelihood value from the original catalogue. Although only a small difference in the ellipticity dispersion is brought about by lensing, this is summed over 19400 galaxies when calculating $\ell$ !

Note that changing the value assumed for the background source redshift, $z$, only has a marginal effect on the best-fit NFW parameters: changing $z$ by 0.25 results in an approximately $5-10 \%$ change in the $M_{200}$ value inferred from the best-fit values of $r_{200}$ and $c$. This indicates that our analysis is not too badly affected by the lack of redshifts for the background sources. However, we hope to obtain photometric redshifts for many of the background sources in order to obtain as accurate a result as possible. Assuming an $\Omega=0.3, \Lambda=0.7$ cosmology changes the mass estimate by $\approx 5 \%$. Again, this mass estimate is consistent (2.5\% difference) with that of Clowe \& Schneider (2001).

An important point to note is that the difference in the projected mass $M_{\Sigma}$ of A1689 within $R=r_{200}$ for the bestfit SIS, power-law and NFW models is less than $20 \%$. This means that adopting a particular parameterised model does not lead to a severe error in the mass estimate.

To conclude, we have applied the shear method discussed in SKE and in KS to the spectacular lensing cluster Abell 1689. The algorithm requires only a few seconds of cpu time to obtain best-fit parameterised models. Our results for the best-fit $\sigma_{1 \mathrm{D}}$ for the SIS and $M_{200}$ for the NFW profile are consistent with standard $\chi^{2}$ fitting; one main advantage of the maximum likelihood method is that galaxies need not be binned. We are very encouraged by the performance of the method, which will be applied to a larger sample of clusters in due course.

Acknowledgements. We would like to thank Simon White for kindly reading the manuscript, and an anonymous referee for many helpful comments. This work was supported by the TMR Network "Gravitational Lensing: New Constraints on Cosmology and the Distribution of Dark Matter" of the EC under contract No. ERBFMRX-CT97-0172.

\section{References}

Allen, S. W. 1998, MNRAS, 296, 392

Bartelmann, M. 1996, A\&A, 313, 697

Bartelmann, M., \& Schneider, P. 2001, Phys. Rep., 340, 291 
Clowe, D., \& Schneider, P. 2001, A\&A, 379, 384

Fontana, A., D'odorico, S., Fosbury, R., et al. 1999, A\&A, 343, L19

Girardi, M., Fadda, D., Escalera, E., et al. 1997, ApJ, 490, 56

Gray, M. E., Ellis, R. S., Refregier, A., et al. 2000, MNRAS, 318,573

King, L. J., \& Schneider, P. 2001, A\&A, 369, 1

King, L. J., Schneider, P., \& Springel, V. 2001, A\&A, 378, 748

Kormann, R., Schneider, P., \& Bartelmann, M. 1994, A\&A, 284, 285

Mellier, Y. 1999, ARA\&A, 37, 127

Miralda-Escudé, J., \& Babul, A. 1995, ApJ, 449, 18

Navarro, J. F., Frenk, C. S., \& White, S. D. M. 1996, ApJ, 462, 563
Navarro, J. F., Frenk, C. S., \& White, S. D. M. 1997, ApJ, 490, 493

Press, W. H., Teukolsky, S. A., Vetterling, W. T., \& Flannery, B. P. 1992, in Numerical recipes in FORTRAN, The art of scientific computing (Cambridge: University Press), 2nd ed.

Schneider, P., Ehlers, J., \& Falco, E. E. 1992, Gravitational lenses (Springer: New York) (SEF)

Schneider, P., King, L. J., \& Erben, T. 2000, A\&A, 353, 41

Schramm, T., \& Kayser, R. 1995, A\&A, 289, L5

Seitz, C., \& Schneider, P. 1997, A\&A, 318, 687

Teague, P. F., Carter, D., \& Gray, P. M. 1990, ApJS, 72, 715

Wright, C. O., \& Brainerd, T. G. 2000, ApJ, 534, 34 